\title{
Casticin Induces Human Glioma Cell Death through Apoptosis and Mitotic Arrest
}

\author{
Enyu Liu Yongqin Kuang Weiqi He Xuemin Xing Jianwen Gu \\ Department of Neurosurgery, General Hospital of People's Liberation Army Chengdu Military Region, \\ Chengdu
}

\author{
Key Words \\ Casticin • Glioma $・$ Cell cycle arrest $•$ Caspase-3 $•$ p53
}

\begin{abstract}
Background: Malignant gliomas are the leading cause of morbidity and mortality in brain and central nervous system tumors. Recently, casticin has drawn wide attention to its critical role in tumor progression. However, the effect of casticin on glioma remains undefined. Methods: Following treatment with casticin, cell viability, apoptosis, and cell cycle arrest were examined in U251 glioma cells. Additionally, the involved molecular mechanism was assessed by western blotting and flow cytometry. Results: Casticin triggered an obvious dose-dependent decrease in U251, U87 and U373 glioma cell viability, and the growth inhibitory effect of casticin was correlated with cell cycle arrest and cell apoptosis. Further mechanistic analysis indicated that casticin induced G2/M phase arrest by attenuating the polymerization of tubulin. Furthermore, striking apoptosis was also confirmed, accompanied by the up-regulation of caspase-3, p53 and proapoptotic protein Bax. These effects were absent when the caspase inhibitor Z-VADfmk or p53 inhibitor PFT $\alpha$ were applied, suggesting that casticin could trigger cell apoptosis in a caspase- 3 and p53-dependent manner. Conclusion: These findings provide a prominent insight into how casticin abrogates the pathogenesis of glioma, and support its potential clinical prospect for further development of anti-brain cancer therapy.
\end{abstract}

Copyright (C) 2013 S. Karger AG, Basel

\section{Introduction}

Glioma is considered to be the most lethal form of brain and central nervous system tumors, and more than $60 \%$ of malignant brain tumors are glioma [1, 2]. It is generally believed that these gliomas are characterized by high rates of proliferation, migration and invasion. Although tremendous advances in the treatment of glioma have been made, 
Liu/Kuang/He/Xing/Gu: Casticin Induces Human Glioma Cells Death

including surgery, radiation therapy, and chemotherapy. They only have modest impact on patient survival. A median survival rate of less than one year after diagnosis is still a undisputed fact $[3,4]$. Recently, several natural anti-cancer drugs have drawn more attention as potential agents for adjuvant brain tumor therapy $[5,6]$.

Natural plant compounds, such as flavonoids, possess a range of anti-tumor activities, anti-inflammatory, and anti-thrombotic activities [7, 8]. As one of these compounds, casticin has attracted much attention due to the role of traditional Chinese medicine in antiinflammatory and anti-cancer development [5]. Casticin is a major polymethoxyflavone from Vitex rotundifolia and can exert multiple biological and pharmacological activities in many diseases $[5,9]$. Numerous studies have suggested that casticin is a promising candidate for treating cancer due to its roles in the growth, cell cycle arrest, and apoptosis of cancer cells including cervical cancer, hepatocellular carcinoma, pancreatic carcinoma, and so on [8-10]. In hepatocellular carcinoma, casticin can aggravate cell apoptosis via up-regulating the protein level of death receptor (DR) 5 [9]. It is widely accepted that anti-cancer agents can disturb cell cycle progression in a special phase and induce cell death [7,11]. Further studies have shown that casticin possesses its growth inhibitory effect on pancreatic cancer cell cycle arrest at the G2/M phase, as well as induces apoptosis by down-regulation of antiapoptotic protein Bcl-2 [8]. Furthermore, casticin regulates the generation of reactive oxygen species (ROS) and sustains the activation of c-Jun N-terminal Kinase (JNK) to mediate HeLa cell apoptosis [10]. Although many studies have been reported on the role of casticin in anti-tumor pathogenesis, its anti-tumor action on glioma and the corresponding molecular mechanism have not been reported.

Consequently, the aim of this study was to investigate the effect of casticin on human glioma, and its growth inhibitory effect on glioblastoma cells was examined. In addition, cell cycle at G2/M phase and cell apoptosis was also discussed here. What we did in this research will provide an insight into the anti-tumor effect of casticin on glioma.

\section{Materials and Methods}

\section{Antibodies and reagents}

Unless otherwise specified, all substances were obtained from Sigma-Aldrich (St Louis, MO, USA). Casticin was purchased from Tauto Biotech Co Ltd. (Shanghai, China). General caspase inhibitor (z-VAD-fmk) and p53 inhibitor (PFT $\alpha$ ) were purchased from Beyotime institute of Technology (Shanghai, China). The polyclonal antibodies against human $\mathrm{p} 53$, caspase-3, Bax and tubulin were from Santa Cruz Biotechnology (Santa Cruz, CA). Antibodies against P21waf1, cyclin B1 and CDK1 were purchased from Cell Signaling Technology (China). Peroxidase-conjugated goat anti-mouse antibody was from Dako Cytomation (Glostrup, Glostrup, Denmark).

\section{Cell culture and treatment}

Human glioma cells U251, U87 and U373 were obtained from the American Type Culture Collection (ATCC, Rockville, MD, USA). Cells were maintained in Dulbecco's Modified Eagle's Medium (DMEM) containing $10 \%$ fetal bovine serum, $100 \mu \mathrm{g} / \mathrm{mL}$ streptomycin and penicillin at $37^{\circ} \mathrm{C}$ with $5 \% \mathrm{CO} 2$, as described previously [7]. Casticin was dissolved in dimethyl sulfoxide (DMSO) with a final DMSO concentration of 1\% before use. Cells were pre-treated with $20 \mu \mathrm{M}$ z-VAD-fmk (general caspase inhibitor) or PFT $\alpha$ (p53 inhibitor) for $24 \mathrm{~h}$, and then followed with various concentrations of prepared-casticin or DMSO. Cells treated with DMSO were used as control.

\section{Proliferation assays}

Cell proliferation was assessed by MTT assay. After pretreatment with different doses of casticin for $24 \mathrm{~h}$, about $20 \mu \mathrm{L}$ of MTT reagent $(5 \mathrm{mg} / \mathrm{mL}$ ) was added to cells, which were incubated for a further $4 \mathrm{~h}$ at $37^{\circ} \mathrm{C}$. The supernatant was replaced with $200 \mu \mathrm{L}$ isopropanol to dissolve and formazan production and the absorbance was measured at $595 \mathrm{~nm}$ with micro-ELISA reader (Bio-Rad, Hercules, CA). The results were expressed as percentage of growth inhibition. 
Liu/Kuang/He/Xing/Gu: Casticin Induces Human Glioma Cells Death

Quantification of live and dead cells

In order to quantify the live/dead cells, the fluorescent probes calcein AM and PI were used. Following treatment with casticin, cells were washed three times with PBS, and then cultured with PBS solution consisting of $2 \mu \mathrm{M}$ calcein AM and $4 \mu \mathrm{M}$ PI in the dark for $20 \mathrm{~min}$. Due to the permeable ability on cell membrane, calcein AM was used to stain viable cells, whereas PI was used to label dead cells. The fluorescence of calcein AM and PI was detected by flow cytometry (Becton Dickinson, San Jose, CA).

\section{Apoptosis analysis}

To quantitatively assess the rate of apoptosis, annexin V-propidium iodide (AV-PI) staining was conducted. Following incubation, an equal number of cells were lysed with lysis buffer (10 mM Tris, $10 \mathrm{mM}$ EDTA, $0.5 \%$ Triton X-100, pH 7.5), rinsed with PBS, and resuspended in $200 \mu \mathrm{L}$ of binding buffer including 5 $\mu \mathrm{L}$ FITC-conjugated annexin V following the manufacturer's instructions (Beyotime, Shanghai, China). After incubation in the dark for $10 \mathrm{~min}$, cells were labeled with PI. Finally, the samples were analyzed through flow cytometry, and the results were expressed as a percentage of total cells counted.

Cell cycle assay

A cell cycle assay was performed according to the method previously mentioned [12]. Briefly, after harvesting by trypsinization, cells were washed with PBS, and then fixed in $70 \%$ ethanol at $4{ }^{\circ} \mathrm{C}$ overnight. Cells were then marked with propidium iodide (PI) for $30 \mathrm{~min}$ at room temperature in PBS containing 50 $\mathrm{mg} / \mathrm{mL}$ PI and $1 \mathrm{mg} / \mathrm{mL}$ RNase A. The DNA content was analyzed by flow cytometry.

\section{DNA fragmentation by Hoechst staining}

To highlight the characteristic morphological changes of apoptosis in U251 cells, the harvested cells were washed with PBS, then stained with DNA-specific fluorescent dye Hoechst 33258 (Molecular Probes, Inc., Eugene, OR) for $0.5 \mathrm{~h}$ at $37^{\circ} \mathrm{C}$. After centrifugation, cells were rinsed three times with PBS, and then resuspended in PBS solution. Morphological changes in U251 cells were observed under a fluorescence microscope. Each experiment was repeated three times.

\section{Western blotting}

After washing three times with PBS, cells were dissociated and total protein extracts of U251 cells were prepared using RIPA lysis buffer (Beyotime, Nantong, China). Following the centrifugation, protein concentrations were detected by the BCA assay (Pierce, Rockford, IL). For western blotting, $100 \mu \mathrm{L}$ of protein was electrophoresed by SDS-polyacrylamide gel electrophoresis, and then transferred onto a polyvinylidene difluoride (PVDF) membrane (Pharmacia, Piscataway, NJ). After blocking with buffer containing 5\% nonfat dry milk in Tris-buffered saline with Tween $(50 \mathrm{mmol} / \mathrm{L}$ Tris, $150 \mathrm{mmol} / \mathrm{L} \mathrm{NaCl}, 0.1 \%$ Tween-20, pH 7.4) at $4^{\circ} \mathrm{C}$ overnight, nitrocellulose membrane was incubated with p53 (1:1000), Bax (1:300), caspase-3 (1:600), tubulin (1:500), cyclin B1 (1:300), p21waf1 (1:500) and Cdk1 (1:300) primary antibodies in 5\% defatted milk for $1 \mathrm{~h}$. After washing, HRP-conjugated secondary antibodies were added at room temperature for 1 $h$. The bound antibodies were visualized using the LumiGLo reagent (Pierce) and the levels of each protein relative to that of $\beta$-actin were analyzed.

\section{Statistical analysis}

All results are shown as mean \pm SEM. Data were analyzed using SPSS 11.0. A typical image from at least three similar experiments was presented. Statistical analysis was performed by an independent Student t-test. $P<0.05$ was considered statistically significant.

\section{Results}

Casticin abrogated glioma cell viability in a dose-dependent manner

To assess the effect of casticin on cell viability in human glioma cells, various concentrations of casticin were used. The extent of cell viability was determined after 24 $\mathrm{h}$ by MTT assay. As shown in Fig. 1A, casticin abrogated the growth of U251 glioma cells in an obvious dose-dependent manner. When cells were treated with a low concentration of 


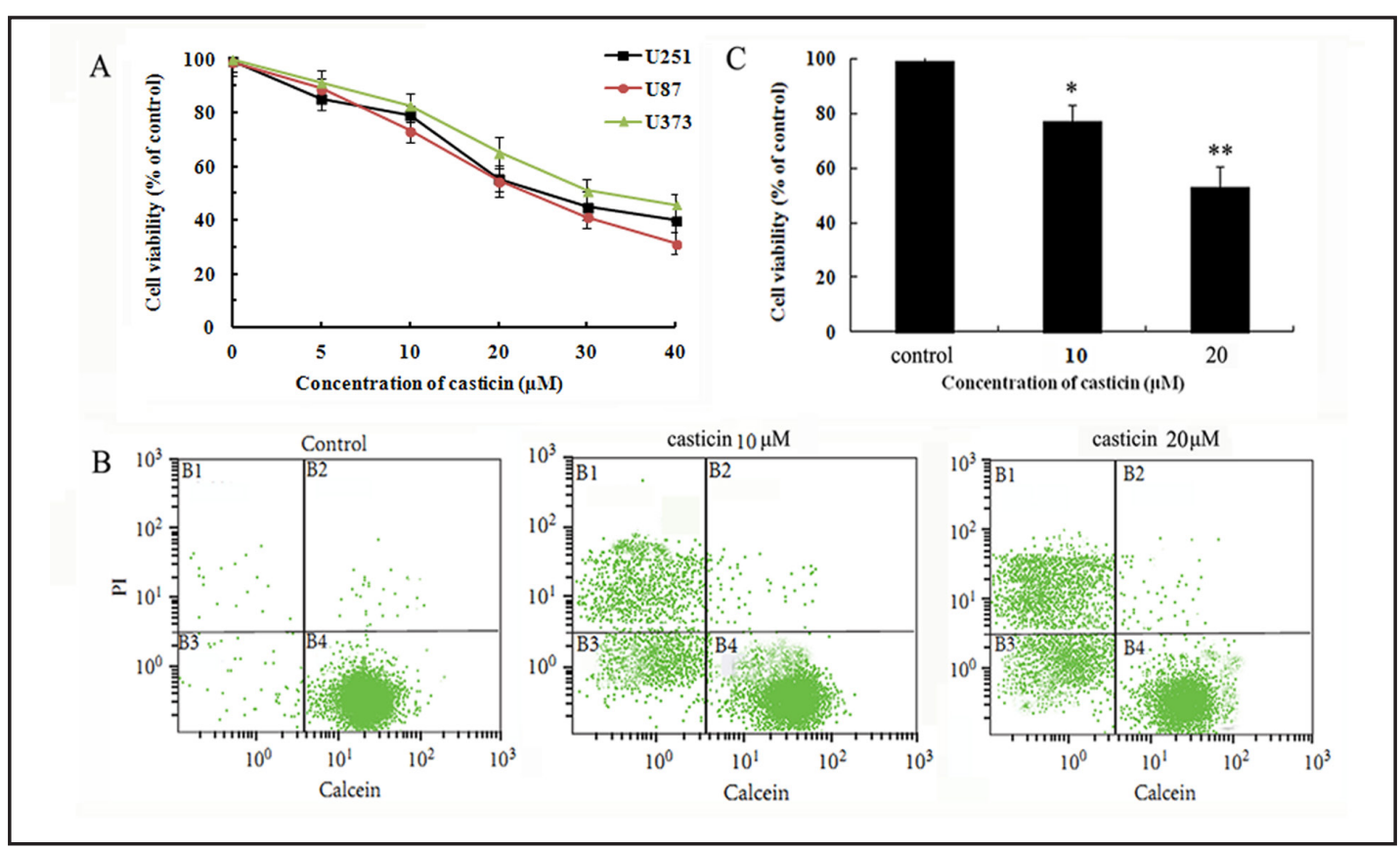

Fig. 1. Effect of casticin on glioma cell viability. (A) U251, U87 and U373 cells were treated with the indicated doses of casticin for $24 \mathrm{~h}$. Cell viability was tested using MTT assay. (B) Following treatment with DMSO (control) or casticin, cells were incubated with $2 \mu \mathrm{M}$ calcein and $4 \mu \mathrm{M}$ PI in the dark for 20 min to determine live/dead cells. All the cells were analyzed using flow cytometry. (C) Quantification of live and dead cells, data are shown as mean \pm SEM. ${ }^{*} \mathrm{P}<0.05 .{ }^{*} \mathrm{P}<0.01$.

casticin, only a slight growth inhibitory effect was observed. However, significant inhibition rate of $22.9 \%$ was confirmed at $10 \mu \mathrm{M}$, and IC50 was $20 \mu \mathrm{M}$. Consistent with the effect of casticin on U251 cells, casticin also triggered a dose-dependent decrease of cell viability in U87 and U373 cells. In addition, treatment with $40 \mu \mathrm{M}$ casticin induced an obvious downregulation of cell viability in U87 cells, compared with $20 \mu \mathrm{M}$ groups. To further confirm the inhibitor effect of casticin on glioma cell, we performed the live/dead cells analysis by fluorescent probes calcein AM and PI. Compared with the control group, abundant fluorescent signals of PI were determined at $20 \mu \mathrm{M}$ casticin-treated group in regions B1 and B3 as well as reduced calcein AM signals in region B4, suggesting that fewer live cells were detected when treated with casticin (Fig. 1B). Quantification analysis showed that the viability of U251 glioma cells stimulated with $10 \mu \mathrm{M}$ and $20 \mu \mathrm{M}$ casticin was $78.1 \%$ and $53.2 \%$, respectively, compared with the control group (Fig. 1C). Taken together, these results indicated that casticin attenuated dose-dependently cell viability.

\section{Casticin enhanced U251 cell apoptosis}

Cell apoptosis is characterized with DNA fragmentation and loss of plasma membrane asymmetry [13]. To investigate whether cell apoptosis is correlated with cell growth inhibition by casticin, we assessed early apoptosis using Hoechst 33258 staining to observe morphological changes in nucleus. After treatment with casticin, an obvious increase in nuclear shrinkage and DNA fragmentation was observed, suggesting that casticin dosedependently induced cell apoptosis by disrupting the nuclear morphology (Fig. 2A). The similar effect on cell apoptosis was further confirmed by Annexin V-FITC and PI staining. Following treatment with $10 \mu \mathrm{M}$ casticin, the apoptotic rate was $19.86 \%$, and a higher apoptotic ratio was observed in $20 \mu \mathrm{M}$ casticin-treated cells (Fig. 2B). All of these results revealed that casticin induced cell apoptosis in a dose-dependent manner. 
Fig. 2. Casticin induced dose-dependently glioma cell apoptosis. (A) After stimulated with DMSO or 10 and $20 \mu \mathrm{M}$ casticin, Hoechst 32258 was added to observe nuclear morphology changes in U251 cells under a fluorescence microscope. (B) Flow cytometry assay of cell apoptosis in U251 cells. Cells were stained with Annexin V/PI. The apoptotic rates were analyzed via flow cytometry. Data shown are mean \pm SEM. ${ }^{*} \mathrm{P}<0.05$. ${ }^{*} \mathrm{P}<0.01$.
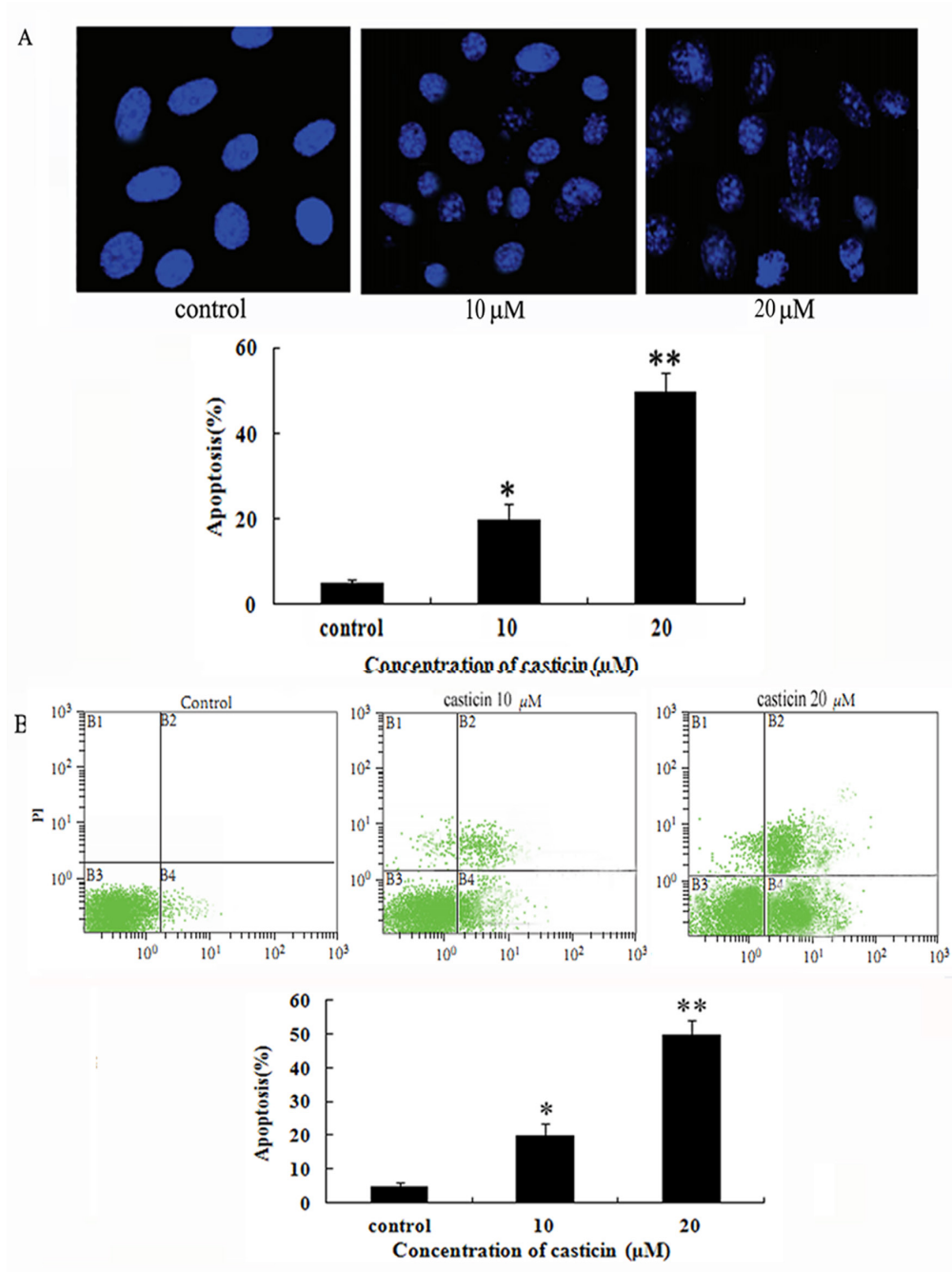

Casticin induced G2/M phase arrest in U251 glioma cells

It is well known that casticin can induce G2/M phase arrest in several tumor cells [14]. Whether an analogous effect of casticin may occur in glioma U251 cells is still unclear. To address this question, cell cycle profile was analyzed by PI staining and flow cytometry assay. As shown in Fig. 3A, treatment with high doses of casticin resulted in the accumulation of cells at G2/M phase. The percentage of cells in the G2/M phase gradually increased from $10.2 \%$ to $28.2 \%$ and $49.8 \%$ after treatment with $10 \mu \mathrm{M}$ and $20 \mu \mathrm{M}$ casticin separately, concomitant with a corresponding decrease in the G0/G1 and S phase (Fig. 3B).

\section{Casticin triggered mitotic phase arrest}

Many anticancer compounds possess tubulin-interacting properties and eventually result in mitotic arrest [15]. To determine whether the inhibitory effect of tubulin polymerization is involved in $\mathrm{G} 2 / \mathrm{M}$ phase arrest, the expression of polymeric tubulin was assessed by western blotting. A clear dose-dependent decrease in tubulin polymerization was demonstrated in U251 glioma cells treated with casticin, indicating that casticin significantly down-regulated polymeric tubulin expression (Fig. 4). It is well known that Cdks, cyclins, and Cdk inhibitors (CKIs) including P21waf1 exert an pivotal role in controlling cell cycle progression $[11,16]$. Therefore, the expression levels of these proteins were discussed during the process of casticin-induced apoptosis via the mitotic phase arrest. The activation of the cyclin B1/Cdk1 complex is vital in the transition from G2 to M phase. As shown in 
Fig. 3. Effect of casticin on cell cycle in U251 glioma cells. Cells were pretreated with, or without $20 \mu \mathrm{M} \mathrm{z}$-VAD-fmk (general caspase inhibitor), and then stimulated with the indicated concentrations of casticin. After washing with PBS, cells were stained with a solution including $50 \mu \mathrm{g} / \mathrm{mL}$ PI in the dark. Flow cytometry was used to assess cell cycle phase distribution. ${ }^{*} \mathrm{P}<0.05 .{ }^{* *} \mathrm{P}<0.01$.

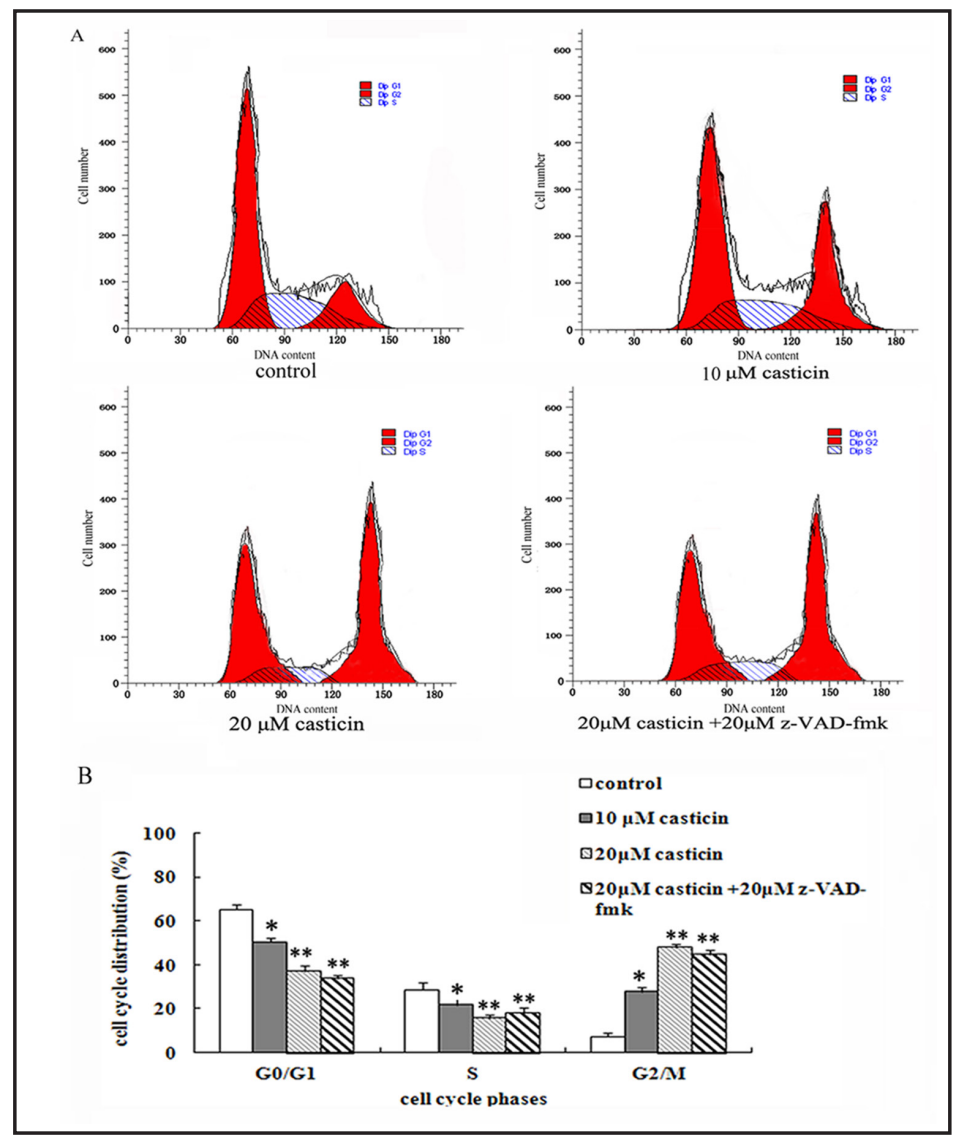

Fig. 4. Effect of casticin on cell cycle regulatory proteins. U251 cells were treated with the indicated concentration of casticin for 24 h. Following harvesting of these cells, the protein expression level of polymeric tubulin, p21, cyclin B1 and Cdk1 were demonstrated by western blotting. ${ }^{*} \mathrm{P}<0.05$.

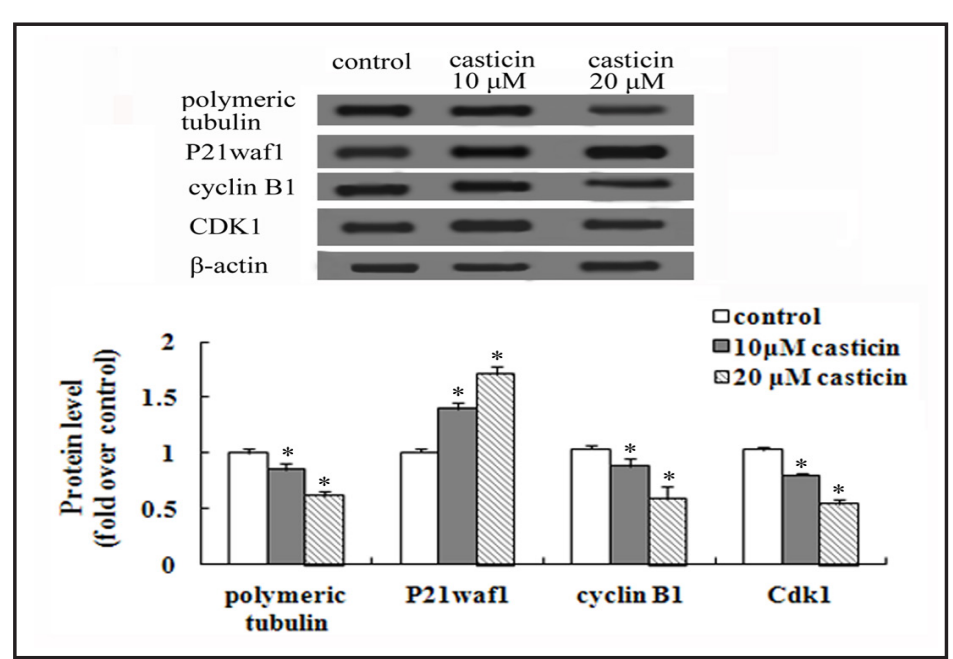

Fig. 4, casticin clearly down-regulated the expression of cyclin B1 as well as Cdk1 whereas it up-regulated P21 waf1 expression, which could inhibit the polymerization of tubulin. So these results suggested that casticin triggered mitotic arrest at the G2/M phase by augmenting tubulin disorganization.

The activation of the caspase-3 and p53 pathway were accounted for casticin-induced apoptosis of U251 glioma cells

It is generally believed that p53 pathway is one of the most powerful tumor suppressor genes. The fact that casticin could induce glioma cell apoptosis has been demonstrated above. 


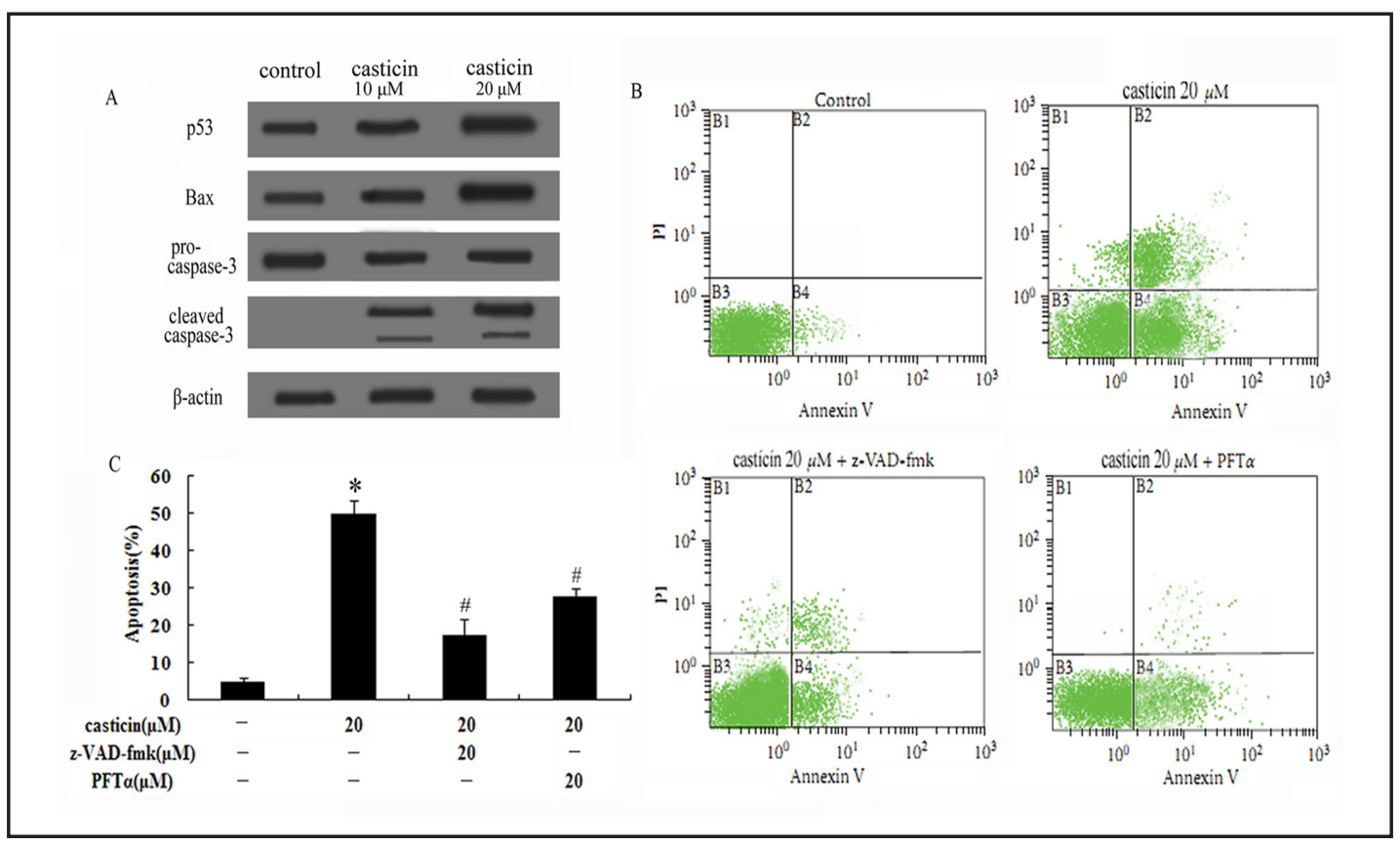

Fig. 5. Casticin induced cell apoptosis in a p53- and caspase-3-dependent manner. Before stimulation with the indicated dose of casticin, U251 cells were pretreated with $20 \mu \mathrm{M} \mathrm{z-VAD-fmk} \mathrm{or} \mathrm{PFT} \alpha$ (p53 inhibitor). The protein levels of p53, Bax and caspase-3 were analyzed by western blotting (A). After staining with Annexin V/PI, flow cytometry was used to measure the apoptotic rate (B). The quantitative data are shown as mean $\pm \mathrm{SEM}(\mathrm{C}) .{ }^{*} \mathrm{P}<0.05$ versus control group. \#\#P<0.05 versus casticin-treated cells.

However, the precise molecular mechanism of action remains unclear. Hence, we attempted to link p53 to casticin-induced cell apoptosis mechanistically. Cells were thus stimulated with various doses of casticin, and western blotting was carried out to assess the expression levels of p53. As shown in Fig. 5A, casticin dramatically increased the expression of p53 in a dosedependent manner. In addition, Bax, a proapoptotic protein that is a key downstream gene in the p53 pathway, was also significantly up-regulated as the gradual increase of casticin concentration. When pretreated with the p53 inhibitor PFT $\alpha$, less apoptotic signaling was detected compared with casticin-treated group (Fig. 5B). Additionally, quantitative analysis indicated that cell apoptotic rate was obviously decreased from $49.72 \%$ to $27.59 \%$ indicating that casticin could induce cell apoptosis via the p53 pathway in glioma cells (Fig. 5C).

Caspase- 3 has been confirmed to execute the apoptotic response in cells, and its cleavage is a hallmark in the process of cell death. To further elucidate the apoptotic mechanism, possible changes in capase- 3 expression was also measured in this study. Western blotting analysis detected the cleavage of caspase- 3 in casticin-treated group (Fig. 5A). Moreover, pretreatment with a general caspase inhibitor, z-VAD-fmk, remarkablely down-regulated the apoptotic rate from $49.72 \%$ to $17.23 \%$ (Fig. 5B and Fig. 5C), which suggested that caspase-3 was involved in the casticin-triggered glioma cell apoptosis.

\section{Casticin-induced apoptosis was not responsible for cell cycle arrest}

To further confirm the correlation between casticin-induced apoptosis and cell cycle arrest, the general caspase inhibitor z-VAD-fmk was used to perform an analysis of cell apoptosis and cell cycle arrest. As shown in Fig. 3, treatment with casticin significantly increased the apoptotic rate, whereas this up-regulation failed following pretreatment with z-VAD-fmk (Fig. 5B). Furthermore, pretreatment with z-VAD-fmk elicited little effect on 
Liu/Kuang/He/Xing/Gu: Casticin Induces Human Glioma Cells Death

cell cycle arrest (Fig. 3). These results revealed that pretreatment with caspase inhibitor strikingly abrogated cell apoptosis but not attenuate mitotic arrest, indicating that casticininduced cell cycle arrest in an apoptosis-independent manner.

\section{Discussion}

Primary brain tumors are inherently serious and life-threatening because of their high morbidity and mortality. Gliomas are ranked as one of the most common brain tumors, and account for $30 \%$ of all brain and central nervous system tumors [17]. Since ideal chemotherapeutic agents not only kill cancer cells but also normal cells, the poor selectivity for tumors mean that they have a limited ability to cure cancer [18]. Recently, an increasing amount of attention has been given to the natural plant compound of casticin, due to its broad spectrum of cytotoxicity towards different cancer cells $[8,9]$. It has been documented that casticin is implicated in the growth inhibition of breast cancer, pancreatic carcinoma, hepatocellular carcinoma and epidermoid cancer $[8,9,14,19]$. Casticin was therefore investigated for its potential therapeutic role in glioma. However, the effect of casticin on glioma cells remains poorly elucidated. In this study, we first investigated the inhibitory effect of casticin on the growth of U251 glioblastoma cells and its precise molecular mechanism involved.

Cell cycle arrest and apoptosis are well known two major causes of growth arrest [20]. Many antitumor drugs achieve their effect by abrogating cell cycle progression at a special checkpoint [7, 11, 21]. For example, curcumin exerts its inhibitory effect on glioma cell growth and proliferation via induction of cell cycle arrest at the G2/M phase [7]. Accordingly, to characterize the inhibitory effect of casticin on glioma cells, the apoptosis and cell cycle were investigated. In this study, we have confirmed that casticin strikingly dampened cell cycle arrest at G2/M phase in a dose-dependent manner, which is consistent with previous reports that casticin arrested the cell cycle at G2/M phase in pancreatic carcinoma cells [8]. Tubulin elicits a pivotal role in the progression of cell division and the maintenance of cell shape $[22,23]$. To further corroborate the molecular mechanism underlying casticininduced G2/M arrest, the expression of polymeric tubulin was discussed by western blotting. An obvious decrease in polymeric tubulin was validated in casticin-treated cells, indicating that casticin impeded cell cycle at mitotic phase. In addition, casticin dose-dependently increased the expression of cell cycle progression inhibitory protein $\mathrm{p} 21$, which would lead to tubulin disorganization. Furthermore, the activity of cyclin B1/Cdk1 was significantly down-regulated in casticin-treated U251 glioma cells. Therefore, these results suggest that casticin induced cell death by mitotic arrest.

It is well known that many anti-tumor agents exert their growth inhibition effect on malignant cells by inducing apoptosis [9, 10, 24]. Apoptosis, a form of programmed cell death, is characterized by DNA fragmentation, loss of plasma membrane asymmetry, and activation of proteases $[13,25,26]$. In this study, obvious DNA fragment and morphological changes were observed in casticin-treated glioma cells. Furthermore, flow cytometry analysis demonstrated that casticin elevated the apoptotic rate of glioma cells in a dose-dependent manner. These results indicate that casticin-induced apoptosis is associated with cell death. However, how casticin induces apoptosis requires further study.

As a tumor suppressor protein, p53 plays a prominent part in the regulation of cell apoptosis [27]. The up-regulation of p53 expression is related to the induction of cell apoptosis by the activity of proapoptotic protein Bax [28]. Caspase-3 has been corroborated as a critical mediator of programmed cell death, and activated caspase plays an important role in the apoptotic response inside the cells [29,30]. Whether casticin-induced cell apoptosis is correlated with the activity of p53 and caspase- 3 remains unclear. To address this question, the expression of p53 and caspase- 3 was analyzed by western blotting. After treatment with casticin, p53 expression levels were notably up-regulated. This increased effect failed to 
Liu/Kuang/He/Xing/Gu: Casticin Induces Human Glioma Cells Death

continue when pre-treated with p53 inhibitor PFT $\alpha$. As an important downstream target of p53, the expression of Bax was also strikingly increased in U251 cells treated with casticin, which is in line with previous studies showing that casticin increased the expression of Bax [8]. Simultaneously, the expression levels of activated caspase-3 were significantly reinforced following treatment with casticin. After blocking the activity of caspase- 3 with the specific caspase inhibitor z-VAD-fmk, cell apoptosis was dramatically attenuated. Accordingly, we can conclude that casticin induce cell death in part via p53 and caspase-3-mediated apoptotic pathways.

In conclusion, our research investigated for a potential therapeutic role of casticin in glioma. In this study, casticin abrogated the growth of U251 glioma cells by arresting the cell cycle at G2/M phase and consequently inducing p53- and caspase-3-depedent apoptosis. These findings support a prominent insight into how casticin exerts its protective effect by impeding the pathogenesis of glioma. Thus, casticin may become a potential lead compound for future development of anti-glioma therapy.

\section{Conflict of Intersest}

The authors have no financial conflicts of interest.

\section{References}

1 Yin D, Wakimoto N, Xing H, Lu D, Huynh T, Wang X, Black KL, Koeffler HP: Cucurbitacin b markedly inhibits growth and rapidly affects the cytoskeleton in glioblastoma multiforme. Int J Cancer 2008;123:1364-1375. DeAngelis LM: Brain tumors. N Engl J Med 2001;344:114-123.

$>2$ Fujita M, Zhu X, Sasaki K, Ueda R, Low KL, Pollack IF, Okada H: Inhibition of stat3 promotes the efficacy of adoptive transfer therapy using type- 1 ctls by modulation of the immunological microenvironment in a murine intracranial glioma. J Immunol 2008;180:2089-2098.

4 Davies E, Clarke C, Hopkins A: Malignant cerebral glioma-i: Survival, disability, and morbidity after radiotherapy. BMJ 1996;313:1507-1512.

5 Feng X, Zhou Q Liu C, Tao M: Drug screening study using glioma stem-like cells. Mol Med Rep 2012;6:11171120.

6 Kundu P, Mohanty C, Sahoo SK: Antiglioma activity of curcumin loaded lipid nanoparticles and its enhanced bioavailability in brain tissue for effective glioblastoma therapy. Acta Biomater 2012;8:2670-2687.

7 Liu E, Wu J, Cao W, Zhang J, Liu W, Jiang X, Zhang X: Curcumin induces g2/m cell cycle arrest in a p53dependent manner and upregulates ing4 expression in human glioma. J Neurooncol 2007;85:263-270.

8 Ding C, Khan M, Zheng B, Yang J, Zhong L, Ma T: Casticin induces apoptosis and mitotic arrest in pancreatic carcinoma panc-1 cells. Afr J Pharm Pharmacol 2012;6:412-418.

-9 Yang J, Yang Y, Tian L, Sheng XF, Liu F, Cao JG: Casticin-induced apoptosis involves death receptor 5 upregulation in hepatocellular carcinoma cells. World J Gastroenterol 2011;17:4298.

10 Zeng F, Tian L, Liu F, Cao J, Quan M, Sheng X: Induction of apoptosis by casticin in cervical cancer cells: Reactive oxygen species-dependent sustained activation of jun n-terminal kinase. Acta Biochim Biophys Sin 2012;44:442-449.

11 Malumbres M, Barbacid M: Cell cycle, cdks and cancer: A changing paradigm. Nat Rev Cancer 2009;9:153166.

12 Zhang P, Liu W, Zhu C, Yuan X, Li D, Gu W, Ma H, Xie X, Gao T: Silencing of gpnmb by sirna inhibits the formation of melanosomes in melanocytes in a mitf-independent fashion. PloS one 2012;7:e42955.

13 Pope RM: Apoptosis as a therapeutic tool in rheumatoid arthritis. Nat Rev Immunol 2002;2:527-535.

14 Haïdara K, Zamir L, Shi QW, Batist G: The flavonoid casticin has multiple mechanisms of tumor cytotoxicity action. Cancer Lett 2006;242:180-190.

15 Rocha DD, Balgi A, Maia AIV, Pessoa OD, Silveira ER, Costa-Lotufo LV, Roberge M, Pessoa C: Cell cycle arrest through inhibition of tubulin polymerization by withaphysalin $\mathrm{f}$, a bioactive compound isolated. Invest New Drugs 2012;30:959-966. 
16 Choi HJ, Fukui M, Zhu BT: Role of cyclin b1/cdc2 up-regulation in the development of mitotic prometaphase arrest in human breast cancer cells treated with nocodazole. PloS one 2011;6:e24312.

17 Goodenberger MKL, Jenkins RB: Genetics of adult glioma. Cancer Genet 2012;205:613-621.

18 Skeel RT, Khleif S: Handbook of cancer chemotherapy. Lippincott Williams \& Wilkins, 2011.

19 Shen JK, Du H, Yang M, Wang YG, Jin J: Casticin induces leukemic cell death through apoptosis and mitotic catastrophe. Ann Hematol 2009;88:743-752.

20 Khan M, Yu B, Rasul A, Al Shawi A, Yi F, Yang H, Ma T: Jaceosidin induces apoptosis in u87 glioblastoma cells through g2/m phase arrest. Evid Based Complement Alternat Med 2012;2012:703034.

-21 Lam M, Carmichael AR, Griffiths HR: An aqueous extract of fagonia cretica induces DNA damage, cell cycle arrest and apoptosis in breast cancer cells via foxo3a and p53 expression. PloS one 2012;7:e40152.

22 Antonarakis E, Heath E, Posadas E, Yu E, Harrison M, Bruce J, Cho S, Wilding G, Fetterly G, Hangauer D, Kwan MF, Dyster LM, Carducci MA: A phase 2 study of kx2-391, an oral inhibitor of src kinase and tubulin polymerization, in men with bone-metastatic castration-resistant prostate cancer. Cancer Chemother Pharmacol 2013;71:883-892.

23 Prinz H: Recent advances in the field of tubulin polymerization inhibitors. Expert Rev Anticancer Ther 2002;2:695-708.

24 Patacsil D, Osayi S, Tran AT, Saenz F, Yimer L, Shajahan AN, Gokhale PC, Verma M, Clarke R, Chauhan SC: Vitamin e succinate inhibits survivin and induces apoptosis in pancreatic cancer cells. Genes Nutr 2012;7:83-89.

25 Stoehr G, Schaab C, Graumann J, Mann M: A silac-based approach identifies substrates of caspasedependent cleavage upon trail-induced apoptosis. Mol Cell Proteomics 2013;12:1436-1450.

26 Hashimoto Y, Hosoda N, Datta P, Alnemri ES, Hoshino S: Translation termination factor erf3 is targeted for caspase-mediated proteolytic cleavage and degradation during DNA damage-induced apoptosis. Apoptosis 2012;17:1287-1299.

27 Slovackova J, Smarda J, Smardova J: Roscovitine-induced apoptosis of h1299 cells depends on functional status of p53. Neoplasma 2012;59:606-612.

28 Goloudina AR, Mazur SJ, Appella E, Garrido C, Demidov ON: Wip1 sensitizes p53-negative tumors to apoptosis by regulating the bax/bcl-xl ratio. Cell Cycle 2012;11:1883-1887.

29 D'Amelio M, Sheng M, Cecconi F: Caspase-3 in the central nervous system: Beyond apoptosis. Trends Neurosci 2012;35:700-709.

-30 Ying TH, Yang SF, Tsai SJ, Hsieh SC, Huang YC, Bau DT, Hsieh YH: Fisetin induces apoptosis in human cervical cancer hela cells through erk1/2-mediated activation of caspase-8-/caspase-3-dependent pathway. Arch Toxicol 2012;86:263-273. 